\title{
Over-DI dissection may question the value of radiotherapy as a part of an adjuvant programme in high-risk radically resected gastric cancer patients
}

\author{
M Scartozzi', E Galizia', F Graziano ${ }^{2}$, V Catalano ${ }^{3}$, R Berardi', AM Baldelli ${ }^{3}$, E Testa ${ }^{2}$, D Mari ${ }^{4}$, RR Silva $^{4}$ and \\ S Cascinu*,I \\ 'Department of Medical Oncology, Università Politecnica delle Marche-Azienda Ospedaliera Ospedali Riuniti, Ancona, Italy; ${ }^{2}$ Department of Medical \\ Oncology, Ospedale di Urbino, Italy; ${ }^{3}$ Department of Medical Oncology, Azienda Ospedaliera S Salvatore, Pesaro, Italy; ${ }^{4}$ Department of Medical Oncology, \\ Ospedale di Fabriano, Italy
}

The aim of our analysis was to assess retrospectively the effect on local relapse, overall survival (OS) and disease-free survival (DFS) of a limited or an extended lymphadenectomy in radically resected gastric cancer patients. This study was performed in order to identify a subgroup of patients possibly not benefiting from a therapeutic approach such as chemoradiation therapy. We divided our patients into two groups according to lymphadenectomy type: group A for limited ( $<25$ resected lymph nodes) and group B for extended ( $>25$ resected lymph nodes) lymph nodes resection. A total of 418 patients were analysed: tumour stage at diagnosis was pT2-3 pNI -3 M0 in 339 patients and pT3 N0 M0 in 79 patients. Median age at diagnosis was 68 years (range 30-92 years). A total of 306 patients (73.2\%) were in group $A$ and 112 (26.8\%) in group B. The median survival time (OS) for patients in groups $A$ and $B$ was 58.8 and 84.8 months, respectively $(P=0.037 \mathrm{I})$; median DFS was 28.8 months in group $A$ and 59.9 months in group $B$ $(P=0.0027)$. At multivariate analysis, extension within the gastric wall, nodal involvement and the number of resected lymph nodes appeared to affect both OS and DFS. An inadequate lymph nodes resection can affect survival and result in a higher incidence of local relapse, making the latter group of patients optimal candidates for adjuvant chemoradiation.

British Journal of Cancer (2005) 92, I05 I- 1054. doi: I0.1038/sj.bjc.6602468 www.bjcancer.com

Published online 15 March 2005

(C) 2005 Cancer Research UK

Keywords: gastric cancer; lymphadenectomy; adjuvant radiochemotherapy

Although in the last few years the overall incidence of gastric cancer has gradually decreased worldwide, it remains second only to that of lung cancer, with an estimated 755000 new cases diagnosed annually around the world (Jemal et al, 2002). Although many advances have been made in the diagnosis and treatment of gastric cancer, the global outcome for patients diagnosed with this disease is still disappointing with a 5-year survival rate not exceeding $30 \%$ of all cases in Western Countries (Bouvier et al, 2002; Nitti et al, 2003).

In operable gastric cancer, both the extent of surgery and the value of adjuvant treatment remain matter of scientific debate, with surgery still representing the cornerstone of any curative procedure (van de Velde and Peeters, 2003; Hohenberger and Gretschel, 2003). Nevertheless, controversy still exists about the adequate extent of lymphadenectomy, which may be limited to perigastric lymph nodes (D1 dissection) or may include removal of the lymphatic chains along the celiac axis, the common hepatic

* Correspondence: Dr S Cascinu, Clinica di Oncologia Medica, Università Politecnica delle Marche-Azienda Ospedaliera Umberto I, Via Conca, 60020 Ancona, Italy; E-mail: cascinu@yahoo.com

Revised 19 January 2005; accepted 25 January 2005; published online 15 March 2005 and splenic artery and the hilus of the spleen (D2 dissection) (Kim and Karpeh, 2002; Alberts et al, 2003; Nitti et al, 2003).

Although a D2 dissection is the generally accepted surgical procedure in Japan, the debate about the benefits of D1 vs D2 lymph nodes dissection is still ongoing (Bonenkamp et al, 1999; Cuschieri et al, 1999; Davis and Sano, 2001; Lewis et al, 2002; Reis et al, 2002; Sierra et al, 2003).

More recently, a general agreement has been achieved about a so-called over-D1 lymphadenectomy (D1 dissection and retrieval of at least 25 nodes) on the basis of the finding that the probability of accurate assessment of lymph node status increases with the number of nodes resected, with a plateau reached at 20-25 nodes (Marubini et al, 2002).

Although this recommendation addresses to the principle that lymph nodes are regarded as indicators rather than governors of disease, several clinical data also indicated that a lymph nodes resection including more than 25 lymph nodes could have a major impact on the global outcome of patients who underwent apparent radical surgery for gastric cancer (Siewert et al, 1998; Marubini et al, 2002). It has been suggested that this improvement could be mainly due to a reduction in local recurrences (Kim and Karpeh, 2002).

Furthermore, it has been recently reported that a chemoradiotherapy adjuvant approach may improve the outcome of 
radically resected gastric cancer by lowering the incidence of local relapse (Macdonald et al, 2001).

The aim of our analysis was to assess retrospectively the possible effects on local relapse, and consequently on overall survival (OS) and disease-free survival (DFS), of limited or extended lymphadenectomy in a homogeneous group of radically resected gastric cancer patients. This analysis was performed in order to identify a subgroup of patients not likely to benefit from a toxic therapeutic approach such as chemoradiation therapy.

\section{PATIENTS AND METHODS}

\section{Patients selection}

The study population was selected from a central database that includes 765 patients with gastric cancer operated in four different institutions.

At the end of each surgical resection, all nodes were dissected from the specimen by a member of the surgical team. Classification of the $\mathrm{N}$-factor was made according to the numeric system introduced by the fifth TNM (N1: metastases in 1-6 regional lymph nodes; N2: metastases in 7-15 regional lymph nodes; N3: metastases in more than 15 regional lymph nodes) (Sobin and Wittekind, 1997).

A total of 418 patients were eligible for our analysis: 339 patients with pT2-3 pN1-3 M0 (tumour invading muscolaris or serosa (T2-3), lymph node metastasis in one to more than 15 regional lymph nodes $(\mathrm{N} 1-3)$, no distant metastasis (M0)) and 79 patients with pT3 N0 M0.

Our analysis was limited to pT3N0, pT2-3N + tumours since they either represent the largest group of patients candidates to radical surgery and those in which an adjuvant therapy may have a role in improving prognosis.

A dissection of $<25$ or more than 25 lymph nodes was, respectively, classified as limited or extended lymphadenectomy. A cutoff of 25 resected lymph nodes has been arbitrarily chosen according to data reported by Marubini et al (2002), Siewert et al (1998) and Wagner et al (1991). Therefore, we divided our patients into two groups: group A for limited and group B for extended lymph nodes resection. All surgical centres involved presented comparable characteristics for the number of gastric cancer patients seen and the number of surgical procedures performed per year. All surgeons involved had the same scientific background and training.

Follow-up of both groups occurred at 3 months intervals for 2 years, then at 6 months intervals for 3 years and yearly thereafter. Follow-up consisted of physical examination, a complete blood count, chest radiography and CT scanning as clinically indicated. The site and date of first relapse and the date of death were recorded.

\section{Data management and statistical analysis}

Statistical analysis was performed with SAS software version 8.2 for Windows (SAS Institute Inc., Cary, NC, USA).

The association between categorical variables was estimated by $\chi^{2}$ test.

Survival distribution was estimated by the Kaplan-Meier method (Kaplan and Meier, 1958). Significant differences in probability of relapsing between the strata were evaluated by log-rank test.

Cox multiple regression analysis was used to assess the role of lymphadenectomy as prognostic factor adjusted for those variables significant at univariate analysis (Cox, 1972).

Hazard ratios and $95 \%$ confidence intervals were estimated from regression coefficients. A significant level of 0.05 was chosen to assess the statistical significance.
For statistical analysis, OS and DFS were defined, respectively, as the interval between radical surgery and death or last follow-up visit and as the interval between radical surgery and clinical progression or death or last follow-up visit if not progressed.

\section{RESULTS}

A total of 418 patients were eligible for our analysis: 339 patients with pT2 -3 pN1-3 M0 and 79 patients with pT3 N0 M0. A total of 249 patients were males $(59.6 \%)$ and 169 females (40.4\%), and median age at diagnosis was 68 years (range 30-92). A total gastrectomy was performed in 249 patients $(59.6 \%)$, whereas the remaining patients underwent a subtotal resection of the stomach.

A total of 306 patients $(73.2 \%)$ received a limited and 112 (26.8\%) an extended lymph nodes dissection. In all, 210 patients $(50.2 \%)$ were classified as N1, $98(23.5 \%)$ as N2, $31(7.4 \%)$ as N3 and $79(18.9 \%)$ as N0. In the whole group, a median of 18 (range $0-68)$ lymph nodes were resected. A total of 66 patients $(15.8 \%)$ had a pT2 and $352(84.2 \%)$ had a pT3 gastric cancer (Table 1$)$.

With a median follow-up of 25 months (range 1-130 months), we recorded information on the site of the first relapse and these sites were categorised as local or distant (Table 2). Local recurrence occurred in $23 \%$ of cases with $<25$ lymph nodes resected and in $4.7 \%$ of those with $>25$ lymph nodes resected $(P=0.0001)$; however, $37 \%$ of those with $<25$ lymph nodes resected and $24.8 \%$ of those with $>25$ lymph nodes resected had distant relapses $(P=0.12)$.

The median DFS for patients in groups A and B was, respectively, 28.8 and 59.9 months $(P=0.0027)$ (Figure 1). At multivariate analysis, extended lymph node dissection appeared an independent prognostic factor for DFS $(\mathrm{HR}=0.52$, CI $0.36-0.74$, $P=0.0003$ ), which was also influenced by extension within the gastric wall (pT2 vs pT3; HR $=0.41$, CI $0.26-0.63, P=0.0001)$ and nodal involvement ( $\mathrm{N} 0$ vs $\mathrm{N}+; \mathrm{HR}=0.33$, CI $0.21-0.52$, $P=0.0001)$.

The extension of lymphadenectomy also resulted determinant in median survival time (OS) with an OS of 58.8 months for patients

Table I Patients characteristics

\begin{tabular}{lccc}
\hline & Whole group & Group A (\%) & Group B (\%) \\
\hline $\begin{array}{l}\text { Number } \\
\text { Age (range) }\end{array}$ & 418 & $306(73.2)$ & $112(26.7)$ \\
Sex & $68(30-92)$ & $68(30-92)$ & $67(33-87)$ \\
$\quad$ Male & 249 & $189(61.8)$ & $60(53.6)$ \\
$\quad$ Female & 169 & $117(38.2)$ & $52(46.4)$ \\
Gastrectomy & & $155(50.7)$ & $94(84)$ \\
$\quad$ Total & 249 & $151(49.3)$ & $18(16)$ \\
$\quad$ Subtotal & 168 & $53(17.3)$ & $13(11.6)$ \\
PT stage & & $253(82.7)$ & $99(88.4)$ \\
PT2 & 66 & $59(19.3)$ & $20(17.9)$ \\
PT3 & 352 & $165(53.9)$ & $45(40.2)$ \\
pN stage & & $73(23.9)$ & $25(22.3)$ \\
PN0 & 79 & $9(2.9)$ & $22(19.6)$ \\
PN1 & 210 & & \\
PN2 & 98 & & \\
PN3 & 31 & &
\end{tabular}

Table 2 Local and distant relapse

\begin{tabular}{lccc}
\hline $\begin{array}{l}\text { Resected } \\
\text { lymph nodes }\end{array}$ & Patients & $\begin{array}{c}\text { Local } \\
\text { relapse (\%) }\end{array}$ & $\begin{array}{c}\text { Distant } \\
\text { relapse (\%) }\end{array}$ \\
\hline$\leqslant 25$ & 306 & $71(23 \%)$ & $115(37 \%)$ \\
$>25$ & 112 & $5(4.7 \%)$ & $28(24.8 \%)$ \\
$P$-value & & 0.0001 & 0.12 \\
\hline
\end{tabular}




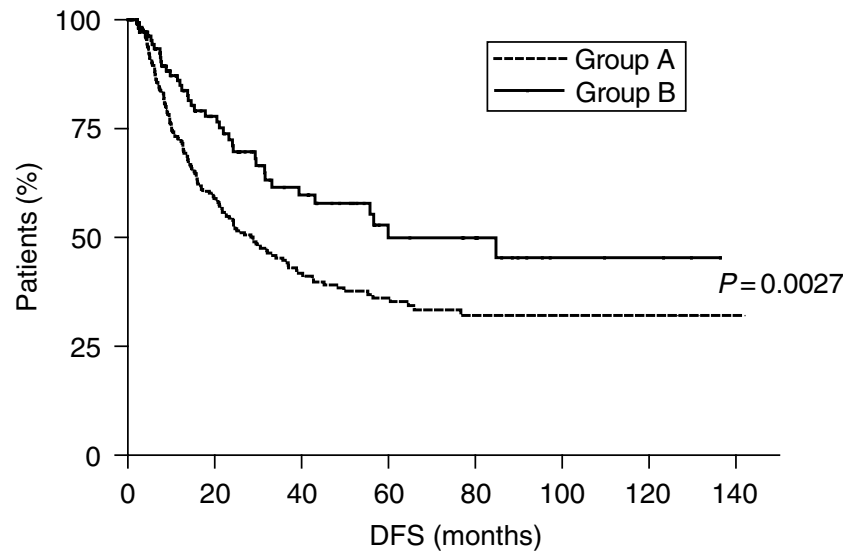

Figure I Disease-free survival of gastric cancer patients undergoing limited ( $\leqslant 25$ lymph nodes resected, group A - - - ) or extended ( $>25$ lymph nodes resected, group $B-$ lymphadenectomy.

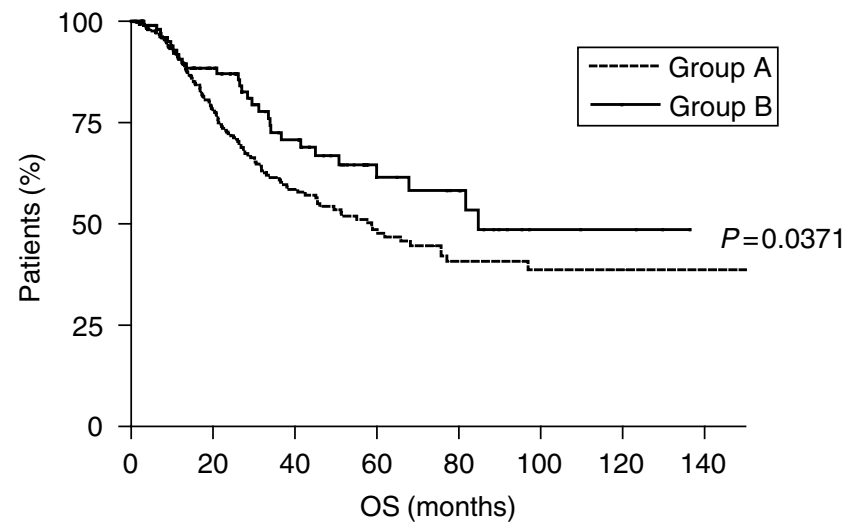

Figure 2 Overall survival of gastric cancer patients undergoing limited ( $\leqslant 25$ lymph nodes resected, group A - - -) or extended (>25 lymph nodes resected, group $\mathrm{B}-$ ) lymphadenectomy.

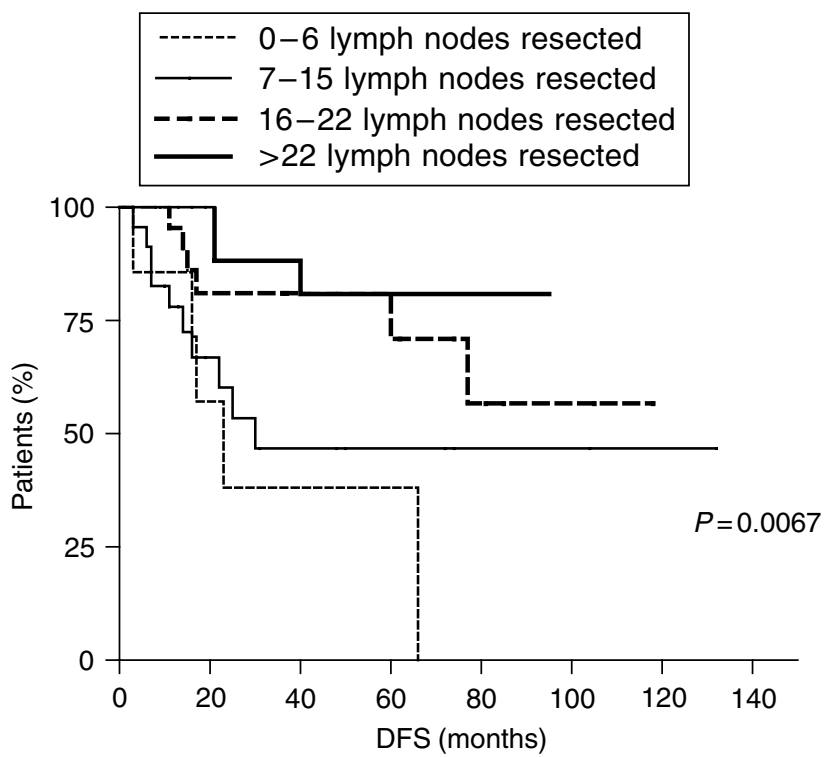

Figure 3 Disease-free survival in lymph nodes-negative gastric cancer patients. Kaplan-Meier curves show that a progressively more extended lymphadenectomy correlates with a progressively better DFS.

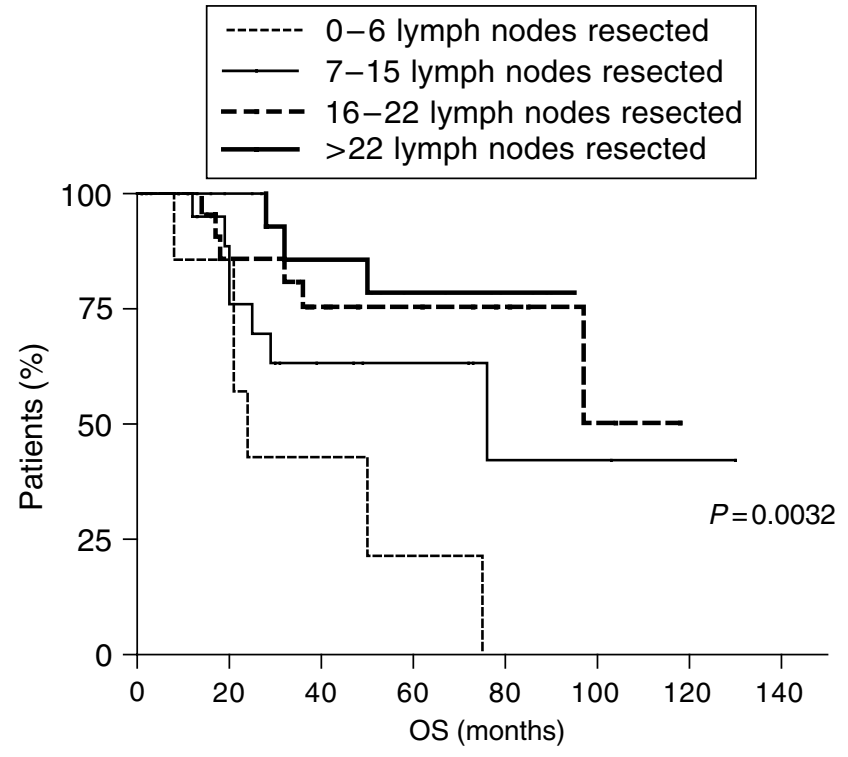

Figure 4 Overall survival in lymph nodes-negative gastric cancer patients. Kaplan-Meier curves show that a progressively more extended lymphadenectomy correlates with a progressively better OS.

in group A and 84.8 months for patients in group B $(P=0.0371)$ (Figure 2).

At multivariate analysis, three variables seemed to influence OS: extension within the gastric wall (pT2 vs pT3; HR $=0.47$, CI 0.29 $0.76, P=0.002$ ), nodal involvement (N0 vs $\mathrm{N}+; \mathrm{HR}=0.46$, CI $0.29-0.74, P=0.0015)$ and the number of resected lymph nodes $(>25$ vs $<25$; HR $=0.59$, CI 0.39-0.89, $P=0.012)$.

These results were confirmed in subgroups analysis, separately considering $\mathrm{N}$-positive and $\mathrm{N}$-negative patients. In fact, among $\mathrm{N}$ positive patients, OS was 51.3 months for those who received limited lymphadenectomy and 84.8 months for those who received extended lymphadenectomy $(P=0.0316)$.

Also, DFS appeared to be influenced by the extension of lymphadenectomy in this latter group of patients, as it was 22.4 months for patients who underwent limited lymphadenectomy and 56.6 months for patients who underwent extended lymphadenectomy $(P=0.0036)$.

In $\mathrm{N}$-negative patients, DFS was progressively affected by a progressively more extended lymph nodes dissection: at a followup of 25 months (range 1-130 months), the DFS of patients with $0-6$ and 7-15 resected lymph nodes was, respectively, 23 and 30 months, while it was not reached for patients with 16-22 and with more than 22 resected lymph nodes. The difference between the four survival curves was statistically significant $(P=0.0067)$ (Figure 3). Similar results were observed for OS: for patients with 0-6 and 7-15 resected lymph nodes, OS was, respectively, 24 and 76 months, while OS was not reached for patients with $16-22$ and with more than 22 resected lymph nodes $(P=0.0032)$ (Figure 4$)$.

\section{DISCUSSION}

One of the main reasons for disease progression in apparently radically resected gastric cancer patients is represented by the frequent occurrence of locoregional relapse (Macdonald et al, 2001; Gunderson, 2002; Park et al, 2003). This high incidence of local relapse provided the rationale for the INT-0116 trial (Macdonald et al, 2001). In fact, this trial showed that a chemoradiotherapy adjuvant approach was able to improve prognosis mainly by lowering the incidence of local relapse (from 
29 to $19 \%$ ); on the contrary, a limited or no effect was seen on metastatic diffusion.

The quality of surgical treatment in this latter study can be considered inadequate, as a substantial proportion of patients (54\%) underwent a D0 resection (no clearance of the lymphatics). On the basis of these considerations, the results of the INT-0116 trial could also be interpreted according to the hypothesis of having treated residual disease by radiochemotherapy in a significant subgroup of patients (Macdonald et al, 2001; Hohenberger and Gretschel, 2003).

Our results seem to confirm these conclusions. In fact, patients undergoing an optimal surgery experienced a local relapse rate as low as $4.7 \%$, whereas this was $21 \%$ in patients receiving a suboptimal surgery. Another important aspect arising from our analysis is once again the lack of effect of optimal surgery on distant metastases. Similar findings were found in the INT-0116 study, when the effects of radiochemotherapy on distant metastases were analysed.

The results we presented in our study seem to go along with data from Siewert et al (1998) and Macdonald et al (2001) (also Hundahl et al, 2002). In these two studies, a survival advantage was suggested for extended lymphadenectomy and in our analysis, the median survival time for patients in groups $\mathrm{A}$ and $\mathrm{B}$ was, respectively, 58.8 and 84.8 months $(P=0.0371)$. An apparent imbalance for the type of gastrectomy (i.e. total $v s$ subtotal gastrectomy in the two groups) could be noticed in our series. In fact, about $84 \%$ of the patients in group A had a total gastrectomy compared to $50.7 \%$ of those in the limited lymphadenectomy group (group B). Therefore, it can be postulated that this latter factor may have affected recurrence and survival. Nevertheless, it has already been demonstrated that the extent of gastrectomy (total or subtotal) has no impact on the global outcome of radically resected gastric cancer patients (Bozzetti et al, 1997).

Although not demonstrated in our data analysis, which did not directly address the role of adjuvant treatment in gastric cancer patients, these findings seem to question the role of adjuvant chemoradiotherapy as a standard of practice in all gastric cancer patients, whereas its role could be that of a partially compensation for an inadequate surgery.

The question remains whether novel and effective chemotherapeutic agents could have a role along with optimal surgery, in order to further improve survival in optimally resected gastric cancer patients.

Therefore, we can hypothesise that patients undergoing suboptimal surgery and consequently at higher risk for local relapse may be suitable for postoperative radiotherapy, whereas those receiving optimal surgery may be candidates to adjuvant chemotherapy as they are considered at higher risk for metastatic progression.

We then believe that our findings could help planning future trials of postoperative management of gastric cancer patients, especially now that new drugs are to be urgently tested in this setting.

\section{REFERENCES}

Alberts SR, Cervantes A, van de Velde CJH (2003) Gastric cancer: epidemiology, pathology and treatment. Ann Oncol 14(S2): ii31-ii36

Bonenkamp JJ, Hermans J, Sasako M, van de Velde CJ (1999) Extended lymph-node dissection for gastric cancer. N Engl J Med 340: 908 - 914

Bouvier AM, Haas O, Piard F, Roignot P, Bonithon-Kopp C, Faivre J (2002) How many nodes must be examined to accurately stage gastric carcinomas? Results from a population based study. Cancer 94: 2862 - 2866

Bozzetti F, Marubini E, Bonfanti G, Miceli R, Piano C, Crose N, Gennari L (1997) Total versus subtotal gastrectomy: surgical morbidity and mortality rates in a multicenter Italian randomized trial. The Italian Gastrointestinal Tumor Study Group. Ann Surg 226: 613-620

Cox DR (1972) Regression models and life-tables. J R Stat Soc 34: 187-220

Cuschieri A, Weeden S, Fielding J, Bancewicz J, Craven J, Joypaul V, Sydes M, Fayers P (1999) Patient survival after D1 and D2 resections for gastric cancer: long-term results of the MRC randomised surgical trial: Surgical Co-operative Group. Br J Cancer 79: 1522 - 1530

Davis PA, Sano T (2001) The difference in gastric cancer between Japan, USA and Europe: what are the facts? What are the suggestions? Crit Rev Oncol/Hematol 40: $77-94$

Gunderson LL (2002) Gastric cancer-patterns of relapse after surgical resection. Semin Radiat Oncol 12: 150-161

Hohenberger P, Gretschel S (2003) Gastric cancer. Lancet 362: 305-315

Hundahl SA, Macdonald JS, Benedetti J, Fitzsimmons T, Southwest Oncology Group, Gastric Intergroup (2002) Surgical treatment variation in a prospective, randomised trial of chemoradiotherapy in gastric cancer: the effect of undertreatment. Ann Surg Oncol 9: 278-286

Jemal A, Thomas A, Murray T, Thun M (2002) Cancer statistics 2002. CA Cancer J Clin 52: 23-47

Kaplan EL, Meier P (1958) Nonparametric estimation from incomplete observations. J Am Stat Assoc 53: 457-481

Kim HJ, Karpeh MS (2002) Surgical approaches and outcomes in the treatment of gastric cancer. Semin Radiat Oncol 12: 162-169

Lewis WG, Edwards P, Barry JD, Khan S, Dhariwal D, Hodzovic I, Allison MC, Shute K (2002) D2 or not D2? The gastrectomy question. Gastric Cancer 5: $29-34$
Macdonald JS, Smalley SR, Benedetti J, Hundahl SA, Estes NC, Stemmermann GN, Haller DG, Ajani JA, Gunderson LL, Jessup JM Martenson JA (2001) Chemoradiotherapy after surgery compared with surgery alone for adenocarcinoma of the stomach or gastroesophageal junction. $N$ Engl J Med 345: 725 - 730

Marubini E, Bozzetti F, Miceli R, Bonfanti G, Gennari L, The Italian Gastrointestinal Tumour Study Group (2002) Lymphadenectomy in gastric cancer: prognostic role and therapeutic implications. Eur J Surg Oncol 28: $406-412$

Nitti D, Marchet A, Olivieri M, Ambrosi A, Mencarelli R, Farinati F, Belluco C, Lise M (2003) Lymphadenectomy in patients with gastric cancer: a critical review. Tumori 2(Suppl 2): S35-S38

Park SH, Kim DY, Heo JS, Lim DH, Park CK, Lee KW, Choi SH, Sohn TS, Kim S, Noh JH, Kim YI, Park JO, Kim K, Kim WS, Jung CW, Im YH, Lee MH, Park K, Park CH, Kang WK (2003) Postoperative chemoradiotherapy for gastric cancer. Ann Oncol 14: 1373-1377

Reis E, Kama NA, Doganay M, Atli M, Dolapci M (2002) Long-term survival is improved by an extended lymph node dissection in potentially curable gastric cancer. Hepatogastroenterology 49: 1167-1171

Sierra A, Regueira FM, Hernandez-Lizoain JL, Pardo F, Martinez-Gonzalez MA, A-Cienfuegos J (2003) Role of the extended lymphadenectomy in gastric cancer surgery: experience in a single institution. Ann Surg Oncol 10: $219-226$

Siewert JR, Bottcher K, Stein HJ, Roder JD, The German Gastric Carcinoma Study Group (1998) Relevant prognostic factors in gastric cancer. Ten-years results of the German gastric cancer study. Ann Surg 228: $449-461$

Sobin LH, Wittekind CH (eds) (1997) TNM Classification of Malignant Tumors, 5th edition, International Union Against Cancer (UICC) pp 59-65. New York: Wiley

van de Velde CJH, Peeters KCMJ (2003) The gastric cancer treatment controversy. J Clin Oncol 21: 2234-2236, (Editorial)

Wagner P, Ramaswamy A, Ruschoff J, Schmitz-Moormann P, Rothmund M (1991) Lymph node counts in the upper abdomen: anatomical basis for lymphadenectomy in gastric cancer. Br J Surg 78: 825-827 